\title{
Yeasts and circumcision in the male
}

\author{
FIONA DAVIDSON
}

From Westminster Hospital, London

SUMMARY Sixty-six circumcised men and 69 uncircumcised men, both heterosexual and homosexual, had specimens taken from the coronal sulcus and meatus of the penis. Yeasts were isolated at similar rates in both the circumcised $(14 \%)$ and uncircumcised $(17 \%)$ men. The circumcised men had significantly fewer symptoms $(P=0.0058)$. Therefore the female partners of both circumcised and uncircumcised men are exposed to similar rates of yeast infection despite the absence of symptoms in circumcised men. Eighty per cent of the female contacts of yeast-positive men had yeast infection while $32 \%$ of the contacts of yeast-negative men were affected. This difference was statistically significant $(0.05>P>0.025)$. Men with non-specific genital infection seemed more likely to carry yeasts than men with gonorrhoea or normal men.

\section{Introduction}

The source of infection of vulvovaginal candidosis is of great importance as candidal infection is now one of the commonest conditions seen in clinics. Recurrent genital candidosis is at present a difficult problem, and this study is part of a larger project.

Although it has been considered that uncircumcised men are more likely to carry candidal species than circumcised men (Hilton and Warnock, 1975; Curzen, 1976) there is little evidence to support this. This study was designed to investigate the problem.

\section{Methods}

One hundred and thirty-five unselected homosexual and heterosexual men attending the Westminster Hospital's Sexually Transmitted Diseases Clinic in June and July 1975, were questioned and examined. Specimens were taken with polyester sponge swabs (Oates et al., 1971). These swabs were turned in the urethral meatus and rolled around the coronal sulcus in both the circumcised and uncircumcised men. Smears were made for direct microscopical examination and stained by Gram's method, and the swabs cultured for yeasts in Feinberg-Whittington medium at a temperature of $37^{\circ} \mathrm{C}$. The cultures were examined twice during the next 48 hours for yeasts but the isolates were not further identified.

\section{Results}

The results are shown in Table 1 . Twenty $(15 \%)$ of the 135 men carried yeasts. Of these 135 men, 66

Address for reprints: Dr F. Davidson, Senior Registrar, Department of Genitourinary Medicine, Westminster Hospital, Dean Ryle Street, Horseferry Road, London SW1P 2AP

Received for publication 6 October 1976
Table 1 Examination of circumcised and uncircumcised men for carriage of yeasts on the penis

\begin{tabular}{llll}
\hline & Circumcised & $\begin{array}{l}\text { Un- } \\
\text { circumcised }\end{array}$ & Total \\
\hline No. of patients & 66 & 69 & 135 \\
Age range (years) & $19-57$ & $18-60$ & $18-60$ \\
No. of heterosexual patients & 59 & 62 & 121 \\
Positive cultures & 8 & 11 & 19 \\
No. of homosexual patients & 7 & 7 & 14 \\
Positive cultures & 1 & 1 & 2 \\
No. of positive cultures & $9(14 \%)$ & $12(17 \%)$ & $21(15 \%)$ \\
\hline
\end{tabular}

were circumcised. Nine ( $14 \%$ ) of the 66 circumcised men and $11(17 \%)$ of the 69 uncircumcised men carried yeasts. There was no statistical difference between these groups. Seven of the circumcised men and seven of the uncircumcised men were homosexual. Only one of these in each group carried yeasts. The incidence in homosexuals was the same as in heterosexuals.

There was no difference in age range between the two groups.

Only three smears showed yeasts in this series, but no smears were positive if the cultures were negative.

\section{SYMPTOMS}

One of the nine circumcised patients with yeastpositive cultures and nine of the 12 uncircumcised patients with yeast-positive cultures had symptoms, Table 2 . This difference is statistically significant $(P=0.0058)$.

\section{CONTACTS}

The female contacts of 35 men were seen, Table 3 . Eight $(80 \%)$ of the 10 contacts of the yeast-positive 
Table 2 Symptoms in yeast-positive men

\begin{tabular}{lll}
\hline Symptoms & Circumcised & Uncircumcised \\
\hline With & 1 & 9 \\
Without & 8 & 3 \\
& $(\mathrm{P} 0.0058)^{*}$ & \\
\hline
\end{tabular}

*Using Fisher's Exact Method (Fisher, 1958).

Using the $\chi^{2}$ test with Yates's correction: $\chi^{2}=6.05$

For one degree of freedom $\begin{cases}P & \chi^{2} \\ 0.025 & 5.024 \\ 0.010 & 6.635\end{cases}$

Therefore $0.025>\mathrm{P}>0.010$

men harboured yeasts, while only eight $(32 \%)$ of the 25 contacts of men without yeasts were affected. This difference is statistically significant $(0.05>\mathrm{P}>$ 0.025 ). Conversely, the male contacts of yeastpositive women were four times more likely to carry yeasts than the male contacts of yeastnegative women.

\section{OTHER CONDITIONS}

Patients with other infections were included in the study; only one of the circumcised yeast-positive patients had diabetes. He was asymptomatic and had no contact at the time. One uncircumcised yeast-positive patient had recently had antibiotics for recurrent non-specific urethritis. He had marked symptoms and his contact had genital yeast infection. Ten $(20 \%)$ of the 49 men with non-specific urethritis, three $(7 \%)$ of the 42 men with no abnormality, and two $(9 \%)$ of the 19 men with gonorrhoea carried yeasts. Infections found in the female contacts included non-specific infection, gonorrhoea, warts, herpes, and trichomoniasis.

\section{Discussion}

Fifteen per cent of the men examined carried yeasts on the penis. This is similar to the findings in another recent report (Rodin and Kolator, 1976). The fact that the yeast isolation rate was the same in both circumcised and uncircumcised men suggests that the presence or absence of the prepuce has no effect on the carriage of yeasts on the penis. This is

Table 3 Diagnosed yeast infection in female contacts of men surveyed for yeasts

\begin{tabular}{lll}
\hline Men & Yeast-positive & Yeast-negative \\
\hline Yeast-positive & 8 & 2 \\
Yeast-negative & 8 & 17 \\
\hline$\chi^{2}=6.63$ with Yates's correction & $4.8384: 0.05>P>0.025$ \\
For one degree of freedom $\left\{\begin{array}{lll}P & \chi^{2} & \\
0.05 & 3.841 & \\
0.025 & 5.024\end{array}\right.$
\end{tabular}

contrary to previous suggestions (Hilton and Warnock, 1975; Curzen, 1976). It is interesting that men carrying yeasts have significantly fewer symptoms if circumcised, although the carriage rate in the two groups is the same. It could be claimed that asymptomatic, circumcised carriers are very likely to be overlooked as a potential source of infection to women.

It is difficult to persuade asymptomatic men to attend a clinic, and they could therefore be a greater source of infection to their female contacts than uncircumcised men who are more likely to seek help because of their symptoms. In contrast with earlier reports that uncircumcised men carry yeasts (Hilton and Warnock, 1975; Curzen, 1976), these findings indicate that it is more likely to be the circumcised men who spread the infection. Men with yeasts should be treated whether or not they have symptoms. There is no doubt that female contacts of men with yeasts have a high infection rate $(80 \%)$ and they should therefore be examined.

On the other hand the results show that men are four times as likely to be yeast-positive if their consorts are yeast-positive. So all male contacts of women with genital yeast infection ideally should be examined and have coronal and meatal cultures taken. However, $32 \%$ of the female contacts of men without yeasts had yeasts themselves, indicating that there are other reasons for yeast infection in women. The results showed that in this series more men with non-specific infection $(20 \%)$ carried yeasts than men with no abnormalities or with gonorrhoea $(7 \%$ and $9 \%)$.

In this study the species of yeast were not identified but a recent study of women with recurrent genital yeast infection attending the department using the same cultural methods, showed that $84 \%$ were Candida albicans, a figure similar to that obtained by Rodin and Kolator (1976).

I would like to thank Dr J. K. Oates for allowing me to investigate these patients under his care, and I am very grateful to $\mathrm{Mr} \mathrm{N}$. Richardson for his technical assistance.

\section{References}

Curzen, P. (1976). Vaginal discharge. Journal of Maternal and Child Health, 1, 28-30.

Fisher, R. A. (1958). Statistical methods in Research Work. 13th edition, pp. 96 and 97 . Oliver and Boyd: Edinburgh.

Hilton, A. L., and Warnock, D. W. (1975). Vaginal candidiasis and the role of the digestive tract as a source of infection. British Journal of Obstetrics and Gynaecology, 82, 922-926.

Oates, J. K., Selwyn, S., and Breach, M. R. (1971). Polyester sponge swabs to facilitate examination of genital infection in women. British Journal of Venereal Diseases, 47, 289-292.

Rodin, P., and Kolator, B. (1976). Carriage of yeasts on the penis. British Medical Journal, 1, 1123-1124. 